\title{
Reciprocal Communication between Counseling Centers \& BITs on Suicidal Students
}

\author{
By Gregg Elliott, MA, LPC
}

\section{Introduction}

Counselors and psychologists are faced with a myriad of challenges when they consider sharing information with a Behavioral Intervention Team (BIT). Another difficulty can be found in the volume of complex and confounding cases with which mental health professionals and BIT members are faced. Some examples of these include:

- A student has a loud argument with Jesus while alone in his residence hall room and is overheard by people in the residence hall commons area.

- A student Tweets that he's "done with everything" and then disappears.

- An art student destroys her almost-completed sculpture with a mallet, leaving debris scattered throughout the art studio.

Behavioral Intervention Teams tasked with addressing issues of suicide, self-harm, emotional distress, homicide, and alarming behaviors are routinely faced with situations that may or may not be related to a student's mental health. It is, therefore, no surprise that $87 \%$ of Behavioral Intervention Teams include Counseling Center representation (Van Brunt, Sokolow, Lewis \& Schuster, 2012), which is the highest rate of representation for any individual campus group. Having the campus mental health experts on the team is a critical component in accurately assigning threat levels, completing mental health assessments, and in crafting interventions to reduce the risk of self-harm or violence directed at others.

However, getting a campus Counseling Center to agree to participate on the BIT is just the first obstacle that must be overcome. A mental health professional's ability to actively participate in BIT communications is complicated by several factors that are not always completely understood by other BIT members. While there is typically no issue with a counselor, social worker, psychologist, or psychiatrist speaking in the hypothetical or offering general educational information about various mental disorder symptoms, diagnoses, and 
standard treatments and interventions for many of the behaviors with which BITs deal, providing specific information about students who are known to, or in a professional relationship with, the mental health professional is almost always restricted or prohibited.

This paper will focus on the topic of intra-team communications related to suicide and suicidal ideation, as these are among the most common issues BITs encounter. An in-depth analysis of the governing standards of multiple professions and their impact on impediments, both perceived and actual, to free and open communication between Counseling Centers and BITs will be presented. A case study will be used to illustrate how issues of restricted communications can be effectively handled within the BIT.

\section{BIT Communications to the Counseling Center The Family Educational Rights and Privacy Act of 1974 (FERPA)}

FERPA is a Federal law designed to protect the rights of students and their educational records (FERPA, 1974). FERPA provides students 18 years and older (or their parents, if under the age of 18) with the right to inspect and review the student's educational records, the right to request that the educational institution correct or amend the student's record (if it's believed that the record is inaccurate or misleading), and the right to restrict to whom the record can be released.

According to FERPA, the term "education records" is defined as those records that contain information directly related to a student and which are maintained by an educational agency or institution or by a party acting for the agency or institution (1974). In most cases, the records maintained by a BIT would be considered part of the student's education record.

The nine exceptions to FERPA's confidentiality requirements are listed in Table 1. 
Table 1: Exceptions to FERPA

\section{Exceptions to FERPA's Confidentiality Requirements}

- School officials with legitimate educational interest;

- Other schools to which a student is transferring;

- Specified officials for audit or evaluation purposes;

- Appropriate parties in connection with financial aid to a student;

- Organizations conducting certain studies for, or on behalf of, the school;

- Accrediting organizations;

- To comply with a judicial order or lawfully issued subpoena;

- Appropriate officials in cases of health and safety emergencies; and

- State and local authorities, within a juvenile justice system, pursuant to specific State law.

- While not listed by the Department of Education as an official exception, FERPA also allows a student's parents access to the student's education record if the student is recognized as a dependent of the parents; for example, if the parents are claiming the student as a dependent on their income taxes.

Note: FERPA allows educational institutions to disclose the student's records without the student's consent to the identified parties and in the identified circumstances. (FERPA, 1974).

FERPA does not prohibit school officials from disclosing information gained through their own personal observation or first-hand from the student and not from the school's educational record (Whiston, 2013). However, if the school official provides that information to another entity within the institution (like the BIT) which subsequently documents the information, then the information would become part of the student's education record and would be covered by FERPA. Students can voluntarily sign a release of information allowing the institution to share their information in whatever way the student authorizes. 
In the case of a BIT providing information to BIT members, including Counseling Center staff, the communication could qualify under the "school officials with legitimate educational interest" exception. Depending on the student's situation and assigned level of threat, the communication could also qualify under the "appropriate officials in cases of health and safety emergencies" exception.

FERPA was never intended to impede the work of BIT teams in gathering information, ascertaining risk and intervening with students. After the shootings at Virginia Tech in 2007, Margaret Spelling, the US Secretary of Education, distributed a letter and brochure articulating how FERPA enables school officials to disclose education records that include personally identifiable information for the purpose of protecting the health or safety of students or other individuals (2013). This exception to the FERPA restrictions is limited to the period of the emergency and it does not allow for unlimited release. Also, this exception would allow the campus BIT to communicate with entities inside, as well as outside, the institution in cases of a current emergency and for the purpose of protecting the campus and/or the community. Appendix $\mathrm{H}$ of the Report of the Virginia Tech Review Panel (2007) similarly clarifies that under FERPA, educational institutions are allowed to disclose information from education records to the parents of students in situations where a health or safety emergency involves their son or daughter.

\section{Counseling Center Communications to the BIT}

Mental health professionals can communicate with a campus BIT, though their privileged information between client and therapist is more restricted. Their communication about clients

is first and foremost restricted by their codes of ethics and by the laws of their state that govern their profession. Confidentiality laws are in place to protect clients in a professional relationship with the mental health professional, and to protect the information that clients provide within the confines of the professional relationship - a relationship that usually begins when a client signs an informed consent document with the mental health professional. If there is ever a conflict between regulations, the mental health professional should follow the most restrictive requirement. When it comes to confidentiality, FERPA will rarely be the 
restriction most relevant to mental health professionals; the code of ethics and laws of the state will typically provide the most guidance.

There are limited exceptions when a campus mental health professional may not be bound by confidentiality regulations. For example, information that a counselor obtained outside her role as a counselor and about a student who was not in a professional relationship with the counselor would not be covered by counselor confidentiality laws. An example of this would be if the counselor assisted in the training of Resident Assistants on campus and was informed by one of the participants after the training that a different resident was struggling with depression. Since the information was obtained from a third party and outside the bounds of a professional relationship, the information would not be covered by counselor confidentiality laws.

There are a variety of different codes of ethics that apply to different types of mental health practices, depending on the type of state licensure. This paper will focus on the code of ethics of the American Counseling Association, as one example of the many ethical codes that govern clinical staff. The ACA code and other codes for different clinical specialties may be found online here:

- The American Counseling Association Code of Ethics: http://www.counseling.org/Resources/aca-code-of-ethics.pdf

- The American Psychological Association Code of Ethics: http://www.apa.org/ethics/code/principles.pdf

- The American Psychiatric Association Code of Ethics: http://www.psychiatry.org/practice/ethics/resources-standards

- The National Association of Social Workers Code of Ethics: http://www.socialworkers.org/pubs/code/default.asp

\section{The ACA Code of Ethics and Confidentiality}

A representative sample of codes from the ACA Code of Ethics relevant to counselor confidentiality is included in Table 2 . These codes, along with others, would need to be considered before a counselor should make the decision to break a client's confidentiality. 
Confidentiality is a cornerstone of the counseling relationship. If a counselor has cause to breach a client's confidentiality, it can only be in a situation where disclosure is required to protect the client or identified others from serious and foreseeable harm. In these circumstances, the law requires 'minimal disclosure' and these disclosures must be restricted to only essential information.

But even mental health professionals who are well-versed in their codes of ethics can find themselves faced with a situation where the codes don't provide definitive direction. Examples of these situations include situations where a suicidal client also has a terminal illness, and situations where a client with a serious disease (like the HIV virus) is having sexual relations with others without notifying his partners about the disease. In situations where the codes of ethics don't provide definitive direction, additional ethical decision making processes are then needed to help a professional determine the most ethical course of action.

Table 2: Confidentiality and ACA

\section{Confidentiality Codes from the ACA Code of Ethics}

A.1.a Primary Responsibility: The primary responsibility of counselors is to respect the dignity and to promote the welfare of clients.

A.4.a Avoiding Harm: Counselors act to avoid harming their clients, trainees, and research participants and to minimize or to remedy unavoidable or unanticipated harm.

B.1.c Respect for Confidentiality: Counselors do not share confidential information without client consent or without sound legal or ethical justification.

B.1.d Explanation of Limitations: At initiation and throughout the counseling process, counselors inform clients of the limitations of confidentiality and seek to identify foreseeable situations in which confidentiality must be breached.

B.2.a Danger and Legal Requirements: The general requirement that counselors keep information confidential does not apply when disclosure is required to protect clients or identified others from serious and foreseeable harm or when legal requirements demand that confidential information must be revealed. Counselors consult with other professionals when in doubt as to the validity of an exception. 
B.2.d Minimal Disclosure: To the extent possible, clients are informed before confidential information is disclosed and are involved in the disclosure decision-making process. When circumstances require the disclosure of confidential information, only essential information is revealed.

H.1.b Conflicts between Ethics and Laws: If ethical responsibilities conflict with law, regulations, or other governing legal authority, counselors make known their commitment to the ACA Code of Ethics and take steps to resolve the conflict. If the conflict cannot be resolved by such means, counselors may adhere to the requirements of law, regulations, or other governing legal authority.

Note: From the ACA Code of Ethics (ACA, 2005).

One such helpful process is to examine the ethical principles that serve as the foundation for most of the codes of ethics. Welfel (2013) discusses five primary ethical principles:

- Respect for Autonomy: respect for the freedom and dignity of each person.

- Nonmaleficence: the responsibility to ensure that counseling does not harm the client.

- Beneficence: the responsibility to do good for the client.

- Justice: the obligation to act fairly.

- Fidelity: faithfulness to promises made and to the truth.

Mental health professionals faced with an ethical dilemma can weigh the importance and priority of the relevant ethical principles to help achieve increased clarity about the best course of action. Keeping the client's personal information confidential is rooted in the ethical principles of Respect for Autonomy and Nonmaleficence.

Clients can voluntarily permit the counselor to provide specific information to specific persons through signing a release of information (see ACA Code B.1.c in Table 2). This respects the client's autonomy (to have the right to choose with whom their personal information is shared), while potentially providing the counselor a little additional freedom to act with beneficence toward the client. In situations where a client signs a release of information, the counselor should work with the client to make it as specific as possible as to whom the information can be released, what information can be released, and how long the release of information will be in effect (Welfel, 2013).

Another ethical decision-making process mental health professionals can use to navigate ethical dilemmas is Welfel's 10-Step Ethical Decision Making Model (2013). Welfel 
recommends a delineated process for mental health professionals to work through when faced with an ethical dilemma:

1. Develop ethical sensitivity

2. Clarify facts, stakeholders, and the sociocultural context of the case

3. Define the central issues and the available options

4. Refer to professional standards and relevant laws/regulations

5. Search out ethics scholarship

6. Apply ethical principles to the situation

7. Consult with supervisor and respected colleagues

8. Deliberate and decide

9. Inform supervisor, implement and document decision-making process and actions 10. Reflect on the experience (Welfel, 2013, pg. 30)

Working through a model such as Welfel's requires some significant time and commitment. Mental health professionals do not always have the requisite time to complete a model such as this one when confronted with an ethical dilemma. In this case, it is best for the clinician to not wait until the crisis in order to wrestle with the ethical dilemma for the first time. Preparing and exploring ethical dilemmas prior to the conflict is an investment in prevention. Clinicians are encouraged to develop a strong foundation in knowledge of the appropriate code of ethics. They should develop the ability to work with the ethical principles, while utilizing knowledge of the applicable state codes that are critical for mental health professionals in responding quickly to complicated ethical situations.

\section{State Confidentiality Laws}

State confidentiality laws typically provide the most guidance to professionals and are the most restrictive source of direction for counselors considering the need to break a client's confidentiality. When it comes to confidentiality laws, the mental health professional has to follow the unique set of rules and guidelines in his or her home state. 
As such, discussing all of the different state laws is beyond the scope of this article. Colorado will be featured as one representative example to highlight common aspects of state counselor confidentiality laws, with reference to several other states for the purpose of highlighting differences. Ultimately, individual counselors must be responsible for knowing and following the laws in their own state. The National Conference of State Legislatures' website (http://www.ncsl.org/issues-research/health/mental-health-professionals-duty-to-warn.aspx) is an excellent resource for researching these laws.

In the State of Colorado, a counselor's duty to confidentiality exists statutorily in C.R.S. $\S$ 12-43-218. This statute provides that mental health practitioners not disclose, without the consent of the client, any confidential information obtained from the client (Lane, 2012).

The law provides for a number of exceptions to confidentiality, including the counselor's duty to warn (C.R.S. § 13-21-117) where the mental health practitioner is required to warn and protect a specific person or persons from a serious threat of imminent physical violence made by the client. The statute is written vaguely enough to allow for the mental health practitioner to take action to protect the client from her or himself if the client indicated a serious suicidal threat. Many states include the duty to protect/duty to warn statutes within their civil codes, documenting that counselors cannot be held civilly liable for breaking a client's confidentiality if the breach is undertaken per the necessity to protect the client or an identifiable other(s) from harm that would be caused by the client.

Colorado's statute is an example of a mandatory reporting law, whereas other states' laws are considered more permissive. For example, Mississippi's law (Mississippi Code Ann. § 42-21-97) says that when a client has communicated an actual threat of physical violence against a clearly or reasonably identified victim or victims, then the [professional] MAY communicate the threat only to the potential victim or victims, or to a law enforcement agency, or to the parent or guardian of a minor who is identified as a potential victim. In January of this year, New York enacted new legislation moving its law from a permissive to a mandatory duty for mental health professionals to report when they believe a client may pose a danger to themselves or others (National Conference of State Legislatures, 2013). 
Exceptions to confidentiality also vary by the state, so mental health practitioners should know and follow the rules of their home state. The six exceptions to confidentiality as recognized by Colorado law include:

- If the counselor suspects child abuse;

- If the counselor feels there is a threat of the client harming him or herself or someone else;

- If the client becomes unable to take care of her or himself and additional help is required;

- If a court orders the counselor to reveal information, or;

- If there is a Grievance Board inquiry;

- A client can also voluntarily release a counselor to provide information to an identified third party.

Colorado's exceptions to confidentiality are somewhat common among the states. But other exceptions to confidentiality do exist depending on the state. For example, in Florida, mental health professionals are also required to breach a client's confidentiality on the basis of suspecting elder abuse (Florida Statutes, 2012). Ultimately, a mental health professional must be knowledgeable of and must follow the unique set of rules and guidelines in their home state.

Also, of note in the State of Colorado, is HB11-1169, the Higher Education Campus Safety Information Sharing Act, which states:

A Colorado state institution of higher education police department may share information regarding incidents that may pose risks to the campus community with certain designated institution administrators or a person who is the subject of a threat of physical violence. A person providing information in good faith to campus officials is immune from civil liability (American Civil Liberties Union of Colorado, 2011).

While this legislation is specific only to Colorado higher education police departments, it represents a movement toward reducing impediments to campus departments sharing information with each other for the purpose of creating safer campus environments.

\section{Case Study: Sarah H.}

Sarah's roommate brings her to the Counseling Center. Sarah is a 20-year-old junior AfricanAmerican student whose roommates have noticed that she has been distracted and withdrawn for the last two weeks. When one of the roommates questioned Sarah about what's going on, 
she broke down and sobbed uncontrollably, but was unable to tell the roommate exactly what she was struggling with. In interviewing Sarah, the counselor was able to determine that Sarah's parents are divorcing, and that Sarah was raped in a male friend's dorm room two weeks ago while she was intoxicated. Sarah has been struggling with thoughts of "just not wanting to be here anymore" and while these thoughts terrify her, she's been having them consistently and with increasing frequency over the last few days. The counselor completes a suicide assessment and finds that Sarah is experiencing high psychological pain and stress, feels hopeless, and blames herself for the rape. While conceding that she is thinking about suicide, Sarah does not have a plan or a timeframe for killing herself. Sarah identifies her little sister at home as her primary reason for living and can still articulate goals about finishing college and pursuing a career. Sarah feels strongly right now that she doesn't want any formal action or investigation undertaken in response to the rape, but she does agree to meet with the counselor twice weekly to start processing everything with which she's dealing.

Meanwhile, the BIT team convenes and Sarah's name is brought up because one of Sarah's professors expressed similar concerns about Sarah's distraction and withdrawal to the Vice President of Student Affairs. Sarah also has a work-study position on campus with Residence Life and she has missed two shifts of work in the last two weeks, most recently yesterday afternoon. This information was also shared with the BIT.

\section{Discussion}

For the purposes of discussion, assume that Sarah's university is in Colorado and that the counselor meeting with Sarah is an intern from the University's Counselor Education program, working under the Director's license as a Professional Counselor.

This case study is an example of when a counselor may have to make a fairly quick decision about what information to share or not share with the BIT. Suppose that the Vice President of Student Affairs mentions what he knows about Sarah and then the Director of Residence Life mentions what she knows about Sarah. At this point, the BIT is already formulating a picture of how Sarah may be doing based on these two points of information. 
The Counseling Center obviously knows a great deal more about Sarah's situation than anyone else on the BIT. What can the counselor share?

Some key points for the counselor to consider:

- Do Sarah's thoughts about suicide meet the legal threshold (the most restrictive regulation) for the counselor to break her confidentiality?

- What do the ACA Code and the ethical principles contribute to the counselor's decision to break or to not break Sarah's confidentiality?

- Has Sarah signed a release of information that would allow the counselor to share specified information with the BIT?

- Once the counselor shares information with the BIT, that information is no longer bound by counselor confidentiality; it's now bound by FERPA. What are the implications?

In a perfect world, Sarah's counselor would have received training on what constitutes a BIT situation and how to handle those situations. At a minimum, because the counselor is an intern practicing under the license of the director, situations like Sarah's should be immediately staffed with the director or clinical supervisor. The Counseling Center could also have internal protocols that when students share information about suicidal ideation or intention, that the student be advised of the work of the BIT and asked to sign a voluntary release that would allow the Counseling Center to share just the basic information about the student's situation with the BIT. The Counseling Center could even consider standard protocols that every student who goes through an intake be advised of the work of the BIT team and be asked to sign a voluntary release that information could be shared with the BIT if a student reached the threshold of being a student of concern.

If the Counseling Center had these protocols in place and Sarah voluntarily released the Counseling Center to share information about her situation with the BIT, then the question becomes what and how much information should be shared? In Sarah's case, she has already indicated to the counselor that she does not want any formal action or investigation undertaken related to the rape incident. Information that the Counseling Center receives related to sexual assaults is exempt from the mandatory reporting requirements of Title IX. 
When this information is shared with the BIT, it becomes part of the student's educational record and would require BIT members to report it to the Title IX officer so the incident could be investigated. To honor the ethical principles of Nonmaleficence and Respect for Autonomy, the specific information about the rape should probably not be shared with the BIT.

From a BIT perspective, it may be enough for the team to know that Sarah was brought to the Counseling Center by one of her roommates, that Sarah voluntarily participated in an intake and that there is some concern about suicide, but that for the time being Sarah has agreed to participate in counseling twice weekly and the Counseling Center will notify the BIT if Sarah stops coming to counseling or if she takes any other actions against the recommendations of the Counseling Center. This should be enough information for the BIT to start making some decisions about Sarah's threat level and to discuss any other interventions that might be appropriate, while protecting information that Sarah might not want to have shared with the committee and which the committee doesn't need in order to consider her situation.

If Sarah has not already signed a release with the Counseling Center, then the Counseling Center representative will need to make some quick decisions about Sarah's situation as it relates to counselor professional ethics and Colorado state law.

First, the information possessed by Sarah's counselor, assuming it is documented in a counseling case file and kept locked in the counselor's office, and assuming that it is information that is used only in connection with Sarah's treatment, is not covered by FERPA. Should Sarah's counselor, for any reason, choose to share this information with the BIT, then the information would be reviewed by the BIT and covered by FERPA from that point forward. At the point of having to decide if and what information to share with the BIT, FERPA is not the primary regulation that the counselor needs to consider.

Ethically, the counselor needs to breach Sarah's confidentiality when "disclosure is required to protect clients or identified others from serious and foreseeable harm" (ACA, 2005, B.2.a). A case can definitely be made that the harm Sarah is thinking about is serious. It's harder to make a case that a suicide attempt is foreseeable for her at this point. If there were additional risk factors present, such as a prior suicide attempt, ongoing struggles with mental health 
concerns, a current suicide plan, and/or a refusal to connect with a counselor, would Sarah's situation then start to approach the ethical threshold for breaking her confidentiality?

In trying to determine if Sarah meets the legal threshold for "clear and imminent threat of physical violence" to self, the clinician has to rely on the suicide evaluation that's been conducted for the client. Wang, Nyutu and Tran (2012) found that coping mechanisms and a client's reasons for living were critical factors in protecting against suicide for Black college students. This finding is consistent with the research base of the "Assessing \& Managing Suicide Risk (AMSR): Core Competencies for Mental Health Professionals," developed by the Suicide Prevention Resource Center (2008) that strong protective factors reduce the overall risk of suicide for a client. Ultimately, it's left to the counselor's judgment as to if Sarah's reasons for living seem strong enough to reduce her suicide risk in the short-term such that the counselor would not break her confidentiality. A structured suicide assessment tool such as David Jobes' Collaborative Assessment \& Management of Suicide (2006) could also be a critical tool in documenting the suicide risk represented by the client.

From the perspective of Colorado law, we have to meet the threshold of "serious threat of imminent physical violence" (C.R.S. § 13-21-117). Does Sarah's situation constitute a "serious threat"? And, if we believe that Sarah is truly considering suicide as an option for dealing with her current pain, is that suicide attempt "imminent"? Without a current suicide plan, it's difficult to consider Sarah's suicidal thoughts an imminent threat, assuming that Sarah has been honest with the counselor about not having a current plan.

It would be completely plausible though, that three different counselors could come to three different conclusions as to whether or not breaking Sarah's confidentiality might be warranted. What does "threat" mean? What does "serious" mean? When the ACA code says "threat," does that mean exactly the same thing as when the Colorado Revised Statutes says "threat?" In a case of an ethical dilemma, the counselor should put intentional effort into documenting both what decision was ultimately made, as well as the decision making process undertaken in order to make the decision.

Utilization of an ethical decision making model such as Welfel's (2013) can be extremely helpful for mental health professionals faced with an ethical dilemma. Many of those steps are 
reflected in the case study discussion in this article. It would be difficult to complete all of these steps in one's mind when faced with the ethical dilemma at the BIT meeting. The stronger process would be to recognize that dilemmas like this one would occur regularly when the Counseling Center sits on the BIT and to proactively put procedures in place (such as a BIT release protocol) that will help the Counseling Center navigate these situations easier.

\section{Conclusion}

There are a number of considerations that must be made when considering how BITs and Counseling Centers will communicate about specific students and specific student situations. Three of the major considerations are FERPA, the ethical requirements of mental health professionals, and the laws of the state in question. Weighing all of the competing requirements can be complicated and time-intensive and is no guarantee that every mental health professional will come to the same conclusion as to whether or not violating a client's confidentiality is warranted.

Counseling Centers should recognize in advance that communications with the BIT about specific students will represent an ethical dilemma and prepare in advance. A protocol where Counseling Centers request a voluntary release of information from clients that allows

the Counseling Center to share limited information with the campus BIT when warranted could be one way to deal with these types of situations. In the absence of a signed release from the client, mental health professionals should have an ethical decision making model they are comfortable using to help them navigate the specific circumstances of the dilemma with which they're faced.

\section{References}

American Civil Liberties Union of Colorado. (2011). HB 1169: Higher Ed Campus Safety Info Sharing. Retrieved on April 26, 2013 from http://aclu-co.org/bill/hb-1169-higher-edcampus-safety-info-sharing

American Counseling Association. (2005). Code of ethics and standards of practice. Retrieved from http://www.counseling.org/Resources/aca-code-of-ethics.pdf 
Colorado Revised Statutes, § 13-21-117 (2006).

Family Educational Rights and Privacy Act (FERPA). (1974). 20 U.S.C.A. 1232g; 34 CFR Part 99 (West, 1997).

Florida Statutes, § 415.1034 (2012).

Jobes, D.A. (2006). Managing suicidal risk: A collaborative approach. New York: The Guilford Press.

Lane, D.K. (2012). The legal guide for practicing psychotherapy in Colorado, 2012 Ed. Denver, CO: Bradford Publishing Co.

Mississippi Code Ann., § 42-21-97 (2006).

National Conference of State Legislatures. (2013). Mental health professionals' duty to protect/warn. Retrieved on April 22, 2013 from http://www.ncsl.org/issuesresearch/health/mental-health-professionals-duty-to-warn.aspx .

Suicide Prevention Resource Center. (2008). Assessing \& managing suicide risk: Core competencies for mental health professionals (Participant manual). Waltham, MA: Education Development Center, Inc.

Van Brunt, B., Sokolow, B., Lewis, W., \& Schuster, S. (2012). NaBITA Team Survey. http://www.nabita.org.

Virginia Tech Review Panel. (2007). Report of the Virginia Tech Review Panel. Retrieved from http://www.governor.virginia.gov/tempContent/techPanelReport.cfm.

Wang, M.C., Nyutu, P.N., \& Tran, K.K. (2012). Coping, reasons for living, and suicide in Black college students. Journal of Counseling \& Development, 90(4), 459-466.

Welfel, E.R. (2013). Ethics in counseling and psychotherapy: Standards, research and emerging issues, 5th ed. Belmont, CA: Brooks/Cole.

Whiston, S.C. (2013). Principles \& applications of assessment in counseling, 4th ed. Belmont, CA: Brooks/Cole. 